\title{
Fetal Fibronectin Testing in Ontario: Successful Government-Sector Collaboration to Achieve High-Quality and Sustainable System Change
}

Charlotte Moore Hepburn and Marilyn Booth

\begin{abstract}
Ontario's province-wide implementation of fetal fibronectin (fFn) technology, a test to identify women unlikely to deliver within two weeks of presentation with symptoms of preterm labour, is a notable example of evidence-informed system improvement and productive government-sector partnership. Increasing demand for costly, high-risk maternal and newborn care in Ontario hospitals prompted a provincial review. Sector experts identified potentially avoidable maternal admissions and transfers to high-risk units for evaluation of suspected preterm labour as an opportunity for system improvement. Limited access to fFn testing was documented, and expert consensus posited that funding rapid clinical testing to identify women at low risk for preterm delivery would yield a significant return on investment. An expert panel recommended evidence-based clinical guidelines. The government swiftly secured funding and initiated a successful implementation strategy, capitalizing on regional perinatal networks. Amassing clinical and care utilization information, framing the data in a policy-relevant context and partnering sector expertise with ministry capability resulted in this technology being effectively implemented in a complex health system.
\end{abstract}

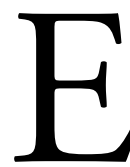
nsuring that the right patient is in the right place at the right time to receive the appropriate level of care is critical to achieving timely, high-quality healthcare for all and to ensuring appropriate resource use across the system. With the demand for costly healthcare services increasing, opportunities that simultaneously achieve an optimal clinical outcome, a superior patient experience and a maximal return on investment are in demand. System change ideas that facilitate improvements in care while enhancing health system sustainability are well received by decision-makers and provide opportunities to strengthen government-sector relations and develop enduring infrastructure to support continuing health system advancement.

The implementation of province-wide fetal fibronectin (fFn) testing, a component of a larger strategy designed to stabilize the high-risk maternal-newborn services sector in Ontario, is an example of a highly successful system change idea. After the development of a compelling clinical and health services business case for new investment in this well-studied technology, the Ontario Ministry of Health and Long-Term Care (MOHLTC) partnered with sector leadership to develop a comprehensive execution strategy. This partnership prompted 
ongoing discussion regarding the capacity for continuous health system improvements for the maternal-child population. This article describes the effective implementation of fFn technology in a complex health system, highlighting how the challenging social and fiscal contexts initiated the dialogue on system change; how the government-sector relationship facilitated an efficient, evidence-based implementation plan; and how enduring regional infrastructure allowed for rapid and costeffective health system improvement.

\section{The Ontario Landscape}

Preterm birth rates in Canada have increased from $6.4 \%$ in 1981 to $8.2 \%$ in 2004 (Public Health Agency of Canada 2008) due to the combined pressures of increases in maternal age, rates of multiple births (both spontaneous and as a result of assisted reproduction; Millar et al. 1992) and rates of obstetrical intervention (Joseph et al. 2002). Accompanied by population growth, this changing epidemiology has challenged the capacity of high-risk maternal and newborn centres to meet this rising demand. Over the past decade, more and more patients were sent out of the region, out of the province and, in some cases, out of the country to receive care that should have been provided close to home. These high-risk transfers peaked in 2007 and captivated media attention with a series of high-profile newspaper articles detailing the lack of available high-risk maternal and neonatal care in Canada (Priest 2007). In this environment, the Ontario MOHLTC commissioned a review of the capacity of Level III neonatal intensive care units (NICUs) in Ontario (Agnew Peckham 2007).

\section{Ontario's Level III NICU Capacity Review}

The Ontario Level III NICU capacity review clearly detailed a shortage of total available bassinets and staff available to operate them. This shortage was considered responsible for not only the high number of out-of-region and out-of-country transfers but also the high occupancy rates that restricted the system's ability to accommodate surges. In addition to documenting the need for investment to increase total capacity, the review identified opportunities to maximize the appropriate use of and/ or decrease demand for these highly specialized and expensive services. The implementation of province-wide cervico-vaginal fFn testing was highlighted as one strategy for maximizing the appropriate use of high-risk obstetrical beds.

\section{fFn Testing}

Cervico-vaginal fFn testing is indicated for women with symptoms of threatened preterm birth prior to advanced cervical dilation. The absence of $\mathrm{fFn}$ is a strong indicator that preterm birth is unlikely to occur. More specifically, a negative test confers a more than $95 \%$ likelihood that the woman will remain "undelivered" for the subsequent 14 days (Leitich and
Kaider 2003; Malak et al. 1996; Peaceman et al. 1997). This information is critically important to enabling an evidenceinformed decision to admit, transfer to a higher level of care or discharge a woman presenting with threatened preterm labour. The use of this technology allows women potentially requiring admission to be identified and those who can safely be monitored as outpatients to avoid unnecessary, costly, disruptive and stressful hospitalizations.

\section{During the 15-month study period, over $\$ 200,000$ was saved in avoided air ambulance transfers.}

Prior to the provincial rollout of fFn technology, only half of Ontario's maternity hospitals offered fFn testing (Provincial Council for Children's Health [PCCH] 2009). In a comprehensive survey, with 88 of 99 maternity hospitals responding, the high cost of the test and the need for substantial provider education (to ensure consistent, evidence-based application of the technology) were highlighted as major barriers to the implementation of the testing in the absence of a provincially sponsored initiative. Importantly, a willingness to implement the technology if appropriate funds and training were made available was echoed by the majority (PCCH 2009).

While randomized controlled trials focusing on resource impact are lacking, a growing body of literature suggests that the successful implementation of fFn testing may result in a reduction in the number of avoidable preterm labour admissions, maternal transfers, maternal length of stay and use of tocolytic agents and corticosteroids (Dutta and Norman 2010). While reports of the technology's impact on total cost savings have been mixed (Musaad et al. 2005) and calculations of actual financial savings are difficult to compare due to variation in both methodologies and healthcare funding systems in different jurisdictions, select Canadian centres have published encouraging preliminary results. In one small, Canadian prospective cohort study, not only were the rates of admission and admission durations positively affected following the implementation of fFn technology, but the costs per patient were significantly reduced. With no change in the preterm birth rate, declines occurred in admission rates (from 24.1 to $12.1 \%$ ), lengths of stay (from 5.2 to 0.6 days) and the mean cost per patient with threatened preterm labour (from $\$ 3,666$ to $\$ 581, p<.0001$; Abenhaim et al. 2005). During the 20 -week study, there was an overall reduction in total cost from $\$ 102,660$ to $\$ 26,169$. In a second Canadian study examining the impact of fFn on healthcare use in Nunavut, a retrospective chart review revealed that, during the 15 -month study period, over $\$ 200,000$ was saved in avoided air ambulance transfers, with no false-negative 
or negative clinical results recorded (MacDonald et al. 2007).

In reviewing this literature, it is clear that $\mathrm{fFn}$ technology provides a promising opportunity to reduce reproductive healthcare resource use without compromising maternal or neonatal outcomes. In addition, women and their families experience reduced disruption, decreased stress and fewer lengthy separations when able to avoid unnecessary admissions and transfers. This is particularly the case for women living in rural and remote locations. The simultaneous pursuit of an improved health outcome, an enhanced care experience and reduced healthcare cost represents the Triple Aim approach defined by the Institute for Healthcare Improvement and seen as the foundational direction of a high-value healthcare system (Berwick et al. 2008). Triple Aim requires providers, institutions and policy decision-makers to innovate, generating solutions to healthcare challenges that evolve beyond the "more and more of what we already have" mantra of dated health system stabilization thinking. Finding new ways of delivering health service to our population - recognizing that more is not always better, and that different can stimulate more meaningful, transformative change - is essential as we face the challenge of long-term health system sustainability.

\section{Dialogue between Government and the Provincial Council for Maternal and Child Health}

In March 2008, continuing concerns about the maternalnewborn system in the province led PCCH and MOHLTC to convene a Maternal-Newborn Advisory Committee (M-NAC) to counsel $\mathrm{PCCH}$ on the optimal system of care for mothers and newborns in Ontario. The mandate of M-NAC is to recommend strategies to achieve a coordinated system of maternal and neonatal services sensitive to urban, rural and remote settings. Strategies focus on the following:

- Equitable access to timely, high-quality, evidence-based, family-centred care at the appropriate level for all pregnant women and newborns in Ontario, as close to home as possible

- Equitable access to specialized services optimizing maternal and newborn care

- Leading evidence-based practice and standards of care

- Data and information systems to inform practice and policy and to promote accountability

This action was one of the catalysts for a review and expansion of the mandate and membership of PCCH in order to align with the government's broader strategic discussions about maternal, child and youth health. In the summer of 2008, a task force with government and sector representation recommended the evolution of $\mathrm{PCCH}$ to the Provincial Council for Maternal and Child Health (PCMCH; Figure 1). The council has been given two distinct roles. First, it is to be an expert advisory body, responding to the needs of MOHLTC and other ministries on issues, priorities and strategies for the maternal, child and youth healthcare system in Ontario. Secondly, it is to be a resource to the maternal, child and youth healthcare system designed to support system improvement and to influence how services are delivered across all levels of care.

\section{FIGURE 1. \\ Collaboration between government and the health sector to implement a plan for improvement}

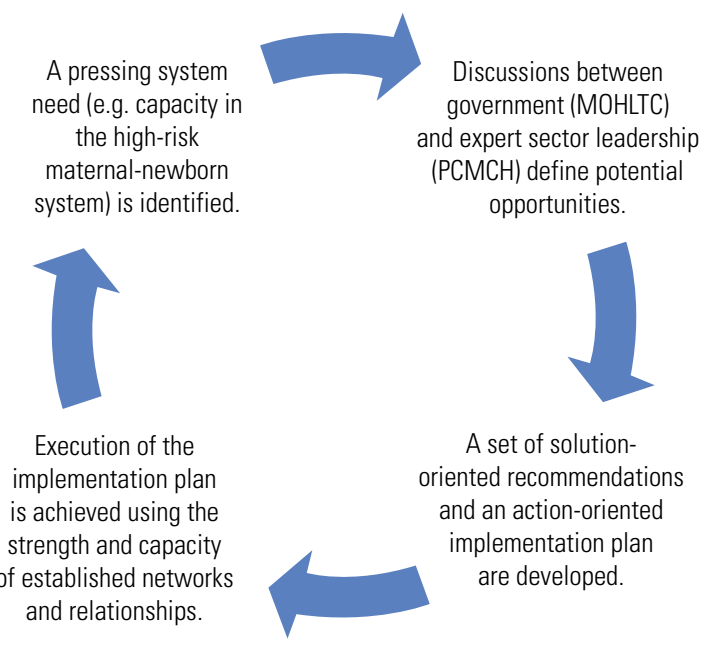

MOHLTC = Ministry of Health and Long-Term Care; $\mathrm{PCMCH}=$ Provincial Council for Maternal and Child Health.

\section{Creation of the Expert Panel}

One of the first initiatives undertaken by M-NAC was to convene an expert panel tasked with recommending an implementation strategy for province-wide fFn testing. Sitting M-NAC members nominated content experts from across the province in the areas of perinatal care, primary care, laboratory services, education and administration to populate the expert panel. Membership selection was based on expertise and reflected diversity of both disciplines and geographical regions. Deliberations began in August 2008.

The panel met three times by teleconference or in person, supplemented by e-mail correspondence. In order to avoid duplication of effort and to leverage the experience of other jurisdictions, fFn testing implementation leads in British Columbia, Alberta and Nova Scotia were interviewed regarding their experience with models for funding, purchasing, distribution, clinician and provider education and system-level evaluation. 
Numerous fFn guidelines from across Ontario, Nova Scotia, Alberta and British Columbia were reviewed. In addition, the 2007 Canadian national fetal fibronectin guidelines (a project of the Canadian Perinatal Programs Coalition [CPPC], which included a comprehensive literature review) were evaluated. With CPPC's permission, their review was updated and information was added from a focused search targeting articles published between 2003 and 2008 and research detailing the impact of $\mathrm{fFn}$ testing on resource use. Furthermore, literature regarding all other available technologies that screen for preterm labour was reviewed and used to inform the technology recommendation of the expert panel (PCCH 2009).

Following deliberations, the expert panel recommended that CPPC's Fetal Fibronectin Guideline for Use in the Management of Preterm Labour (2007) be adopted with modifications to better reflect Ontario's needs and the current literature. These guidelines have become the Ontario standard and are available on the $\mathrm{PCMCH}$ website at www.pcmch.on.ca.

The panel also recommended that MOHLTC support the immediate implementation of $\mathrm{fFn}$ testing, in all Ontario hospitals providing birthing services, through the following actions:

- Promoting the use of $f F n$ testing

- Funding the purchase of analyzers for those Ontario hospitals without this equipment

- Funding test kits for all hospitals providing birthing services

- Supporting the dissemination and implementation of PCMCH's Fetal Fibronectin Guideline for Use in the Management of Preterm Labour (2008)

- Establishing an evaluation plan to determine the impact of fFn testing on resource use and patient outcomes $(\mathrm{PCCH}$ 2009)

One of the challenges in enabling equitable access to $\mathrm{fFn}$ testing is ensuring consistency with the First Nations Inuit Health Branch (FNIHB), the government body responsible for health planning for First Nations and Inuit populations. The expert panel liaised with FNIHB in order to understand the availability of fFn testing in the regions covered by FNIHB and to ensure that FNIBH was aware of the recommended service standards and educational materials created to support the Ontario strategy (PCCH 2009).

The expert panel's report was submitted to MOHLTC in December 2008 and the ministry took immediate steps toward implementation, starting January 2009. Capital funds for analyzer purchases were distributed by March 2009, with accompanying pro-rated funds for the test kits, based on historical volumes. Funding for test-kit supplies was secured for fiscal 2009-2010 onwards. In addition, MOHLTC provided the necessary monies to contract with an active project manager, the Child Health Network for the Greater Toronto Area, to ensure the successful execution of the implementation plan. This network worked with other regional perinatal networks, appointing an $\mathrm{fFn}$ coordinator in each network to assume responsibility for working with hospitals within the geographical region to maximize the uptake of both the test and the supporting evidence-based clinical guidelines.

\section{Recognizing that more is not always better, and that different can stimulate more meaningful, transformative change is essential.}

Strategies that supported the successful implementation included the following:

- A workshop with the appointed regional coordinators to prepare them for their role

- Regular teleconferences to monitor implementation and problem solve any emerging issues

- The development of tools including educational presentations and videos, policy and procedure templates capable of being customized by each hospital, and staff and patient educational materials

- A tracking tool to monitor fFn use at the hospital level

All implementation materials are available on the $\mathrm{PCMCH}$ website.

As of March 1, 2010, 103 of 109 hospital sites were using or indicated their intent to use fFn testing. Of the six hospitals that declined to adopt $\mathrm{fFn}$ testing, most reported a very low level of birthing activity or are in the process of transferring this service out of the institution.

The implementation of fFn testing across Ontario was both effective and efficient as a result of (1) pre-existing communication networks established through the regional networks; (2) the ability to leverage the connections across regional networks for the establishment of a team of regional coordinators to design and implement a roll-out strategy; and (3) dedicated, active project management funded by the government with resources available to support the regional implementation activities.

PCMCH and MOHLTC are committed to ensuring a rigorous evaluation of the impact of making $\mathrm{fFn}$ testing available provincially. An evaluation, funded by the government, is nearing completion.

\section{Conclusions}

Ontario's implementation of fFn testing is an example of effective system change. fFn technology offered an opportunity to improve clinical care quality and the patient care experience while simultaneously enhancing system sustainability. As shown 
through this example, such valuable initiatives can be used to "set the stage" for continued system improvement. In the years to come, it is likely that similar opportunities to meet coincident quality and cost-effectiveness goals will emerge. The swift adoption of these future technologies can be facilitated through vehicles such as $\mathrm{PCMCH}$, an organization with a productive, long-standing relationship with government, able to quickly harness sector leadership and expertise and able to efficiently link with regional network infrastructure to facilitate rapid knowledge translation. HQ

\section{Acknowledgements}

We wish to acknowledge both the financial and in-kind support provided by PCMCH and MOHLTC that enabled the execution of this project. However, we accept that the views expressed herein are those of the authors and do not necessarily reflect the views of our partners. All errors and omissions are entirely our own.

We wish to thank the following individuals for their contributions to the expert panel: Tim Bisschop, Liz Darling, Peter Hutten-Czapski, Moya Johnson, Ann Mitchell, Sarah Newbery, Lucille Perreault, Ann Sprague, Daunett Tucker, Johan Viljoen and Mark Walker.

We wish to acknowledge Alison Quigley and Moya Johnson, of the Child Health Network for the Greater Toronto Area, and the members of the regional perinatal networks for their contribution to the successful implementation of the project.

We also wish to thank Mary Ellen Salenicks, former senior project manager at $\mathrm{PCMCH}$, for her commitment and dedicated support to the expert panel and for her authorship of the expert panel report.

Lastly, we wish to acknowledge Ms. Sherina Macedo, senior policy analyst at the Ontario MOHLTC. Due to her extraordinary commitment, dedication, perseverance and leadership, the Ontario provincial fFn roll-out was a rapid and thorough success.

\section{References}

Abenhaim, H.A., L. Morin and A. Benjamin. 2005. "Does Availability of Fetal Fibronectin Testing in the Management of Threatened Preterm Labour Affect the Utilization of Hospital Resources?" Journal of Obstetricians and Gynaecology of Canada 27(7): 689-94.

Agnew Peckham. 2007. Review of Neonatal Intensive Care Units in Ontario. Toronto, ON: Author.

Berwick, D.M., W.N. Thomas and J. Whittington. 2008. "The Triple Aim: Care, Health and Cost." Health Affairs 27(3): 759-69.

Canadian Perinatal Programs Coalition. 2007. Fetal Fibronection: Guideline for Use in the Managenment of Preterm Labour. <http://rcp. nshealth.ca/sites/default/files/resources-reports/ffn_guideline.pdf $>$.

Dutta, D. and J.E. Norman. 2010. "The Efficacy of Fetal Fibronectin Testing in Minimizing Hospital Admissions, Length of Hospital Stay and Cost Savings in Women Presenting with Symptoms of Preterm Labour." Journal of Obstetricians and Gynaecology 30(8): 768-73.
GTA Child Health Network. 2010. A Workable and Cost-Effective Approach to Provincial Implementation of Fetal Fibronectin Testing: A Review of Highlights and Lessons Learned in Ontario. Toronto: Ontario Ministry of Health and Long-Term Care

Joseph, K.S., K. Demissie and M.S. Krame. 2002. "Trends in Obstetrical Intervention, Stillbirth and Preterm Birth." Seminars in Perinatology 26(4): 250-59.

Leitich, H. and A. Kaider. 2003. "Fetal-Fibronectin - How Useful Is It In the Prediction of Preterm Birth." British Journal of Obstetrics and Gynaecology 110: 66-70.

MacDonald, W.A., M. Bender and A. Saxton. 2007. "Use of Fetal Fibronectin in the Management of Preterm Labour in Nunavut." Alaska Medicine 49(2 Suppl.): 215-17.

Malak, T.M., F. Sizmu, S.C. Bell and D.J. Taylor. 1996. "Fetal Fibronectin in Cervicovaginal Secretions as a Predictor of Preterm Birth." British Journal of Obstetrics and Gynaecology 103(7): 648-53.

Millar, W.J., S. Wadhera and C. Nimrod. 1992. "Multiple Births: Trends and Patterns in Canada, 1974-1990." Health Reports 4(3): 223-50.

Musaad, S.M., C.L. Melson and D.R. Boswell. 2005. "Assessment of the Impact of Introducing Fetal Fibronectin Assay in the Management of Preterm Labour at Middlemore Hospital, New Zealand.” Pathology 37(3): 226-30.

Peaceman, A.M. W.W. Andrews, J.M. Thorp, S.P. Cliver, A. Lukes, J.D. Iams et al. 1997. "Fetal Fibronectin as a Predictor of Preterm Birth in Patients with Symptoms: A Multi-center Trial." American Journal of Obstetrics and Gynecology 177: 13-18.

Priest, L. 2007, July 24. "Canadians Sent to U.S. for Neonatal Care." The Globe and Mail. Provincial Council for Children's Health. 2009. Report of the Provincial Maternal-Newborn Advisory Committee Fetal Fibronectin Testing Work Group. Toronto, ON: Author.

Provincial Council for Maternal and Child Health, 2008. Fetal Fibronectin Guideline for Use in the Management of Preterm Labour, 2008. Accessed August 22, 2011. <http://www.pcmch.on.ca>.

Public Health Agency of Canada. 2008. Canadian Perinatal Health Report-2008 Edition. Ottawa, ON: Author.

\section{About the Authors \\ Charlotte Moore Hepburn, MD, FRCPC, FAAP, is the} medical lead for the Child Health Policy Initiative, University of Toronto Department of Paediatrics, and The Hospital for Sick Children; and a faculty pediatrician in the Division of Paediatric Medicine, University of Toronto Department of Paediatrics and The Hospital for Sick Children, in Toronto, Ontario.

Marilyn Booth, RN, MHSc, is the executive director of the Provincial Council for Maternal and Child Health, in Toronto, Ontario. 